\title{
Assessment of Antioxidant, Antimicrobial, Cytotoxicity and Thrombolytic Potential of Erythrina fusca Lour Grown in Bangladesh
}

\author{
Adeeba Anjum ${ }^{1,2}$, Md. Zakir Sultan ${ }^{3}$, Mohammad Abdullah Al Mansur ${ }^{4}$, \\ Choudhury M. Hasan ${ }^{1}$ and Mohammad A. Rashid ${ }^{1}$
}

${ }^{1}$ Department of Pharmaceutical Chemistry, Faculty of Pharmacy, University of Dhaka, Dhaka-1000, Bangladesh

${ }^{2}$ Department of Pharmacy, University of Rajshahi, Rajshahi-6205, Rajshahi, Bangladesh

${ }^{3}$ Centre for Advanced Research in Sciences (CARS), University of Dhaka, Dhaka-1000, Bangladesh

${ }^{4}$ Institute of National Analytical Research and Service (INARS), Bangladsesh Council of Scientific and Industrial Research (BCSIR), Dr. Kudrat-I-Khuda Road, Dhanmondi, Dhaka-1205, Bangladesh

(Manuscript received: June 2, 2021; Accepted: August 31, 2021; Published (web): November 15, 2021)

\begin{abstract}
The current study was conducted to assess the antioxidant, antimicrobial, cytotoxicity and thrombolytic potential of the leaves and stem bark of Erythrina fusca Lour grown in Bangladesh. For the assessment of antioxidant properties, DPPH scavenging and phosphomolybdenum assays were used. In the DPPH free radical scavenging assay, the chloroform soluble fraction of a methanol extract of leaves of $E$. fusca exhibited the highest free radical scavenging activity with an $\mathrm{IC}_{50}$ value of $5.5 \pm 0.60 \mu \mathrm{g} / \mathrm{ml}$, while the reference standard (ascorbic acid) showed an $\mathrm{IC}_{50}$ value of $5.8 \pm 0.18 \mu \mathrm{g} / \mathrm{ml}$. All the test samples and the standard BHT demonstrated good linear relationship in the phosphomolybdenum assay. It was observed that the test samples contained considerable number of bioactive compounds including total phenolics in the aqueous soluble fraction of the leaves giving the highest $94.02 \pm 1.004 \mathrm{GAE} / 100 \mathrm{~g}$ of dried sample. All test samples exhibited moderate antimicrobial activity against 13 Gram-positive and Gram-negative bacterial strains and 3 fungi, where the chloroform soluble fraction of the stem bark demonstrated the highest inhibition of growth with zone of inhibition of $19.3 \pm 0.21 \mathrm{~mm}$ against Bacillus cereus. All the test samples displayed cytotoxic activity against brine shrimp nauplii having significant $\mathrm{LC}_{50}$ and $\mathrm{LC}_{90}$ values. The chloroform soluble materials demonstrated highest lethality with $\mathrm{LC}_{50}$ value of $1.55 \pm 0.30$ $\mu \mathrm{g} / \mathrm{ml}$ and $\mathrm{LC}_{90} 65.68 \pm 0.23 \mu \mathrm{g} / \mathrm{ml}$. On the other hand, the carbon tetrachloride soluble fraction of the stem bark showed the highest clot lysis $(22.75 \pm 0.59 \%)$ as compared to that $(66.77 \pm 0.61 \%)$ revealed by reference standard, streptokinase. The findings of this study revealed that the leaves and stem bark of E. fusca growing in Bangladesh possesses bioactive compounds but further studies are required to purify and identify them.
\end{abstract}

Key words: Erythrina fusca, antioxidant, free radical scavenging, antimicrobial, cytotoxicity, thrombolytic.

\section{Introduction}

Erythrina is a genus of flowering plants in the pea family, Fabaceae. The generic name is derived from the Greek word (erythros), meaning "red," referring to the flower color of certain species (Gledhill, 2008).
Although some species show pink, purple, orange, white, green, yellow and even coral colors (Stein, 2009; Salma et al., 2017). The genus has 290 species of which Erythrina fusca Lour is known as purple coral tree (Synonym: E. atrosanguinea Ridl.,

Corresponding author: Mohammad A. Rashid; Email: r.pchem@yahoo.com

DOI: https://doi.org/10.3329/bpj.v25i1.57834 
E. glauca Willd., E. ovalifolia Roxb.). The tree is locally known as 'Manya mandar, Kanta mandar or Harikakra'. It is a perennial, medium to large spreading tree $10-15 \mathrm{~m}$ tall with spines $(1-2 \mathrm{~cm})$ (Orwa et al., 2009). E. fusca is the most widespread species in the genus Erythrina that grows in both the Old and New World tropics (Ricardo et al., 1997). In Bangladesh, this plant is distributed in Chittagong and Cox's Bazar and mainly found along the swamps in main land (Flora of Bangladesh).

In various countries different parts of the plant $E$. fusca Lour have been used as traditional medicines. In Thailand, its root, bark and leaves are used as an antipyretic (Dasuki 2001), while the bark and leaves are utilized to relieve inflammation (Widianto 1980). The bark of this plant is used to relieve migraine in Peru (Valkenburg et al., 2001). The seeds are used to cure skin diseases while the inner bark is scraped for dressing fresh wounds in Indonesia (Singapore Botanic Garden, 2013).

A complete literature survey uncovered that most of the parts of the plant have been subjected assays for biological activities like anti-viral, anti-inflammatory, anti-tussive, rheumatism, hematuria, central nervous system depressant, hypotensive and as uterine stimulant (Widianto 1980; Singapore Botanic Garden, 2013; Wasuwat 1967; Russo et al., 1997; Mc Kee et al., 1997; Perry 1980; Ross et al., 1980; Ratnasooriya et al., 1999). Interestingly, the leaves and stem bark have not been subjected to assessment of antioxidant, antimicrobial, cytotoxicity and thrombolytic activities. In continuation of our phytochemical research the crude methanolic extract along with its $n$-hexane, carbon tetrachloride, chloroform and aqueous soluble fractions of the leaves and stem bark of E. fusca were assessed for its antioxidant, antimicrobial, cytotoxicity and thrombolytic activities in the present study.

\section{Materials and Methods}

Collection of plant materials and extraction: Fresh leaves and stem bark of E. fusca were collected from Nandail, Mymensingh District, Bangladesh in
September, 2011 and were authenticated in Bangladesh National Herbarium, where a voucher specimen has been preserved representing this collection (Accession No. DACB-35902). After adequate cleansing, the leaves and stem barks were cut into small pieces, at first air dried, then oven dried at relatively low temperature $\left(30^{\circ} \mathrm{C}\right)$ for 24 hours and finally pulverized into a coarse powder. The powdered leaves and stem bark ( 800 g) was soaked separately in $2.25 \mathrm{~L}$ methanol for seven days, filtered through fresh cotton bed and finally with Whatman No. 1 filter paper, and concentrated by using a rotary evaporator at low temperature $\left(36-40^{\circ} \mathrm{C}\right)$ and reduced pressure. The concentrated crude methanol extracts (CME) of the stem bark and leaves were obtained as $30 \mathrm{~g}$ and $15 \mathrm{~g}$, respectively.

The modified Kupchan partitioning protocol by VanWagenen et al. (1993) was used for the partitioning of an aliquot of the concentrated crude methanol extract (CME) of the stem bark and leaves of E. fusca. The consequent partitionates were dried off to yield $n$-hexane (HSF), carbon tetrachloride (CSF), chloroform (CHSF) and aqueous (AQSF) soluble fractions. The residues were then kept in a cool dry place until further use.

Determination of antioxidant activity: For the determination of antioxidant potential of the crude methanolic extract and its various soluble fractions 2,2-diphenyl-1-picrylhydrazyl (DPPH) free radical scavenging assay as developed by Brand-Williams et al. (1995) was followed.

Total antioxidant capacity: The total antioxidant capacity of the plant extracts was evaluated by the phosphomolybdenum method (Prieto et al., 1999) based on the reduction of Mo (VI) to Mo (V) by the extracts and subsequent formation of a green phosphate-Mo (V) complex in acidic condition.

Total phenolic content: The total phenolic content in the plant samples were determined spectrophotometrically by employing Folin-Ciocalteu reagent as oxidizing agent and gallic acid as the standard for equivalence (Agbor et al., 2014) as developed and described by Skerget et al. (2005). 
Antimicrobial activity: The antimicrobial activity was performed using the agar disc diffusion method (Bauer et al., 1966). Kanamycin (30 $\mu \mathrm{g} / \mathrm{disc})$ and griseofulvin $(20 \mu \mathrm{g} / \mathrm{disc})$ were used as reference standard against antibacterial and antifungal tests, respectively.

Cytotoxicity study: To ascertain the general toxic properties of the plant extractives the method established by Meyer et al., 1982 and Rahman et al., 2008a was employed. This method known as the brine shrimp lethality bioassay, is a useful method which indicates presence of bioactive compounds using dimethyl sulfoxide (DMSO) solutions of plant extractives against Artemia salina in a 1-day in vivo assay. As positive control vincristine sulfate was used. The logarithm of the sample concentration was used to calculate the median lethal concentration $\left(\mathrm{LC}_{50}\right)$.

Thrombolytic activity: The method developed by Prasad et al. (2006) and slightly modified by Kawsar et al. (2011) was used to evaluate the thrombolytic activity of all the test samples. Streptokinase was used as standard.

Statistical analysis: For statistical analysis of all the assays, three replicates of each sample were used and the values are reported as mean \pm SD.

\section{Results and Discussion}

The main objective of our current study was to determine the antioxidant activity and total antioxidant capacity, total phenolic content, assessment the antimicrobial, brine shrimp lethality and thrombolytic activities of the crude methanolic extract of E. fusca as well as its soluble fractions. All the results have been illustrated in Tables 1-5.

The parameter generally used for the measurement of antioxidant activity was determined by the required concentration of the antioxidant to reduce the initial DPPH concentration by $50 \%\left(\mathrm{IC}_{50}\right)$ (Sanchez-Moreno et al., 1998). The $\mathrm{IC}_{50}$ value is inversely proportional to the antioxidant power (Sethupandian et al., 2017). The crude methanolic plant extract along with its modified Kupchan fractions of both the stem bark $\left(\mathrm{IC}_{50} \leq 12.1 \mu \mathrm{g} / \mathrm{ml}\right)$ and leaves $\left(\mathrm{IC}_{50} \leq 19.9 \mu \mathrm{g} / \mathrm{ml}\right)$ displayed significant
DPPH scavenging activity compared to the values obtained for ascorbic acid $\left(\mathrm{IC}_{50}=5.8 \mu \mathrm{g} / \mathrm{ml}\right)$ and tert-butyl-1-hydroxytoluene $\quad(\mathrm{BHT}) \quad\left(\mathrm{IC}_{50}=24.35\right.$ $\mu \mathrm{g} / \mathrm{ml})$ as standards. All results are shown in Table 2 .

Table 1. Modified Kupchan partitionates of the crude methanolic extract of $E$. fusca leaves and stem barks.

\begin{tabular}{lcc}
\hline $\begin{array}{l}\text { Crude extract/ soluble } \\
\text { fractions }\end{array}$ & $\begin{array}{c}\text { Leaves } \\
(\mathrm{g})\end{array}$ & $\begin{array}{c}\text { Stem bark } \\
(\mathrm{g})\end{array}$ \\
\hline $\mathrm{CME}$ & 15 & 30 \\
$\mathrm{HSF}$ & 4.9 & 8.25 \\
$\mathrm{CSF}$ & 1.23 & 2.50 \\
$\mathrm{CHSF}$ & 0.95 & 2.00 \\
$\mathrm{AQSF}$ & 6.7 & 13.1 \\
\hline
\end{tabular}

Phosphomolybdenum assay was used to determine the total antioxidant capacity of all the extractives expressing as the $\mathrm{mg}$ of ascorbic acid per gram of plant extract. The lowest total antioxidant capacity was shown by the chloroform soluble fraction of the leaves $(106.10 \pm 0.89 \mathrm{mg}$ of ascorbic $\mathrm{acid} / \mathrm{g}$ of dried extract) of E. fusca and highest was given by crude methanolic extract of the leaves $(587.66 \pm 0.85 \mathrm{mg}$ of ascorbic acid/g of dried extract) as shown in Table 2. Plant polyphenols are effective as singlet oxygen scavengers, reducing agents and hydrogen atom donors (Karaman et al., 2010; Rice-Evans et al., 1996). That is why, it was logical to determine their total amount in the prepared extracts and fractions of $E$. fusca. Possible participation from other readily oxidized compounds in the plant materials and heterogeneousness of natural phenolics has led to introduction of several methods for determination of total phenolics. In most cases, Folin-Ciocalteu method has been found preferable as compared to the others (Singleton et al., 1999). The amount of total phenolic content varied for the mother extract along with its different soluble fractions of the stem bark and leaves (Table 2) ranging from $8.82 \pm 0.351 \mathrm{mg}$ to $70.17 \mathrm{mg}$ and $11.47 \mathrm{mg}$ to $94.02 \mathrm{mg}$ of Gallic Acid Equivalence (GAE)/100 g of dried extract, respectively. The highest total phenolic content was found from aqueous soluble fraction of the leaves $(94.02 \pm 1.004 \mathrm{mg}$ of GAE/100 $\mathrm{g}$ of dried 
extract) and the lowest in the carbon tetra chloride soluble fraction of the stem bark $(8.82 \pm 0.351 \mathrm{mg}$ of $\mathrm{GAE} / 100 \mathrm{~g}$ of dried extract). The crude methanolic extract along with its four soluble fractions were screened for antimicrobial activity against five
Gram-positive and eight Gram-negative bacteria and three fungi, and the findings are summarized in Table 3. For comparison purposes standard antibiotic discs of kanamycin and griseofulvin were used.

Table 2. Free radical scavenging, ferric reducing capacity, hydroxyl radical scavenging and total antioxidant activities of $E$. fusca.

\begin{tabular}{lccc}
\hline Plant & $\begin{array}{c}\text { DPPH free radical } \\
\text { scavenging activity } \\
\left(\mathrm{IC}_{50} \mu \mathrm{g} / \mathrm{ml}\right)\end{array}$ & $\begin{array}{c}\text { Total antioxidant } \\
\text { capacity (mg of ascorbic } \\
\text { acid/g of dry extract) }\end{array}$ & $\begin{array}{c}\text { Total phenolic content (GAE/100 g } \\
\text { of dry sample) }\end{array}$ \\
\hline Stem bark & $11.8 \pm 0.35$ & & \\
CME & $7.7 \pm 0.95$ & $175.66 \pm 0.63$ & $10.22 \pm 0.505$ \\
HSF & $13.1 \pm 0.75$ & $125.99 \pm 0.29$ & $46.11 \pm 0.463$ \\
CSF & $8.8 \pm 0.95$ & $216.54 \pm 0.47$ & $8.82 \pm 0.351$ \\
CHSF & $12.1 \pm 0.45$ & $415.10 \pm 2.12$ & $26.92 \pm 0.829$ \\
AQSF & & & $70.17 \pm 0.463$ \\
Leaves & $9.9 \pm 0.95$ & $587.66 \pm 0.85$ & $22.13 \pm 0.617$ \\
CME & $7.9 \pm 0.70$ & $142.21 \pm 0.52$ & $11.47 \pm 0.600$ \\
HSF & $19.9 \pm 0.95$ & $106.10 \pm 0.89$ & $12.97 \pm 0.189$ \\
CSF & $5.5 \pm 0.60$ & $260.77 \pm 0.19$ & $30.90 \pm 0.223$ \\
CHSF & $12.6 \pm 0.20$ & $153.77 \pm 0.63$ & $94.02 \pm 1.004$ \\
AQSF & & & - \\
Standard & $5.8 \pm 0.18$ & - & - \\
AA & $24.35 \pm 0.02$ & - & \\
BHT & & & \\
\hline
\end{tabular}

During antimicrobial screening by the disc diffusion assay, all the plant samples of E. fusca at concentration of $400 \mu \mathrm{g} / \mathrm{ml}$ exhibited varying degrees of antimicrobial activity (zone of inhibition $=$ 12.1-19.3 mm) against all the test organisms (Table 3). The highest zone of inhibition was exhibited by the chloroform soluble fraction of the stem bark against B. cereus $(19.3 \mathrm{~mm})$. The manifestation of antibacterial activity of the crude methanolic extract along with its $n$-hexane, carbon tetrachloride, chloroform and aqueous soluble fraction against both Gram-positive and Gram-negative bacteria, and fungi may be indicative of the presence of broad-spectrum antimicrobial compounds. But more investigation is required for proper utilization of this plant as a source of active antimicrobial principle(s).
In the brine shrimp lethality bioassay, cytotoxic compounds generally exhibit significant activity. Because of its simplicity and low cost, this assay can be recommended as a guide for the detection of antitumor and pesticide compounds (Mazid et al., 2008). Also, this bioassay has a good correlation with the human solid tumor cell lines. As there is a correlation between cytotoxicity and activity against the brine shrimp nauplii using plant extracts thus the cytotoxic effect of the plant extracts can be selected for further cell line assay (Manilal et al., 2009). In the case of brine shrimp lethality bioassay, a plot of percentage of the shrimps killed after 24 hours against the logarithm of the sample concentration was used to calculate the median lethal concentration $\left(\mathrm{LC}_{50}\right)$ and $90 \%$ lethal concentration $\left(\mathrm{LC}_{90}\right)$. 
Regression analysis was used to obtain the best-fit The chloroform soluble materials of the stem bark line from the graph. The correlation $\left(\mathrm{R}^{2}\right)$ between exhibited highest lethality with $\mathrm{LC}_{50}$ value of $1.55 \pm$ concentration and mortality was 0.7997 to 0.9880 . $\quad 0.30 \mu \mathrm{g} / \mathrm{ml}$ and $\mathrm{LC}_{90} 65.68 \pm 0.23 \mu \mathrm{g} / \mathrm{ml}$ (Table 4).

Table 3. Antimicrobial activity of stem bark and leaf extracts of $E$. fusca $(400 \mu \mathrm{g} / \mathrm{disc})$, kanamycin $(30 \mu \mathrm{g} / \mathrm{disc})$ and griseofulvin $(20 \mu \mathrm{g} / \mathrm{disc})$.

\begin{tabular}{|c|c|c|c|c|c|c|c|c|c|c|c|}
\hline \multirow{3}{*}{$\begin{array}{l}\text { Test bacteria } \\
\text { Gram-positive } \\
\text { bacteria }\end{array}$} & \multicolumn{11}{|c|}{ Zone of inhibition (mm) } \\
\hline & \multicolumn{5}{|c|}{ Stem bark } & \multicolumn{5}{|c|}{ Leaf } & \multirow{2}{*}{$\frac{\text { PC }}{\text { Kan }}$} \\
\hline & CME & HSF & CSF & CHSF & AQSF & CME & HSF & CSF & CHSF & AQSF & \\
\hline Bacillus cereus & $\begin{array}{c}17.5 \pm \\
0.40\end{array}$ & $\begin{array}{c}16.9 \pm \\
0.75\end{array}$ & $\begin{array}{l}15.2 \pm \\
0.55\end{array}$ & $\begin{array}{c}19.3 \\
\pm 0.21\end{array}$ & $\begin{array}{c}14.2 \pm \\
0.51\end{array}$ & $\begin{array}{c}18.2 \pm \\
0.83\end{array}$ & $\begin{array}{c}17.9 \pm \\
0.36\end{array}$ & $\begin{array}{c}17.4 \pm \\
0.91\end{array}$ & $\begin{array}{l}15.8 \pm \\
0.40\end{array}$ & $\begin{array}{c}17.1 \pm \\
0.72\end{array}$ & $\begin{array}{l}35 \pm \\
0.58\end{array}$ \\
\hline B. megaterium & $\begin{array}{c}18.1 \pm \\
0.68\end{array}$ & $\begin{array}{c}18.6 \pm \\
0.70\end{array}$ & $\begin{array}{c}14.9 \pm \\
0.17\end{array}$ & $\begin{array}{c}12.4 \\
\pm 0.70\end{array}$ & $\begin{array}{l}14.4 \pm \\
0.74\end{array}$ & $\begin{array}{c}17.1 \pm \\
0.32\end{array}$ & $\begin{array}{c}18.1 \pm \\
0.72\end{array}$ & $\begin{array}{c}15.7 \pm \\
0.65\end{array}$ & $\begin{array}{l}17.2 \pm \\
0.72\end{array}$ & $\begin{array}{c}15.4 \pm \\
0.92\end{array}$ & $\begin{array}{l}35 \pm \\
0.58\end{array}$ \\
\hline B. subtilis & $\begin{array}{c}18.4 \pm \\
0.75\end{array}$ & $\begin{array}{c}18.3 \pm \\
0.40\end{array}$ & $\begin{array}{c}16.8 \pm \\
0.36\end{array}$ & $\begin{array}{c}17.6 \\
\pm 0.42\end{array}$ & $\begin{array}{c}14.8 \pm \\
0.31\end{array}$ & $\begin{array}{c}17.5 \pm \\
0.53\end{array}$ & $\begin{array}{c}16.9 \pm \\
0.35\end{array}$ & $\begin{array}{c}14.7 \pm \\
0.35\end{array}$ & $\begin{array}{c}16.1 \pm \\
0.71\end{array}$ & $\begin{array}{c}13.5 \pm \\
0.32\end{array}$ & $\begin{array}{l}36 \pm \\
1.00\end{array}$ \\
\hline Sarcina lutea & $\begin{array}{c}18.3 \pm \\
0.85\end{array}$ & $\begin{array}{l}17.5 \pm \\
0.32\end{array}$ & $\begin{array}{c}16.6 \pm \\
0.31\end{array}$ & $\begin{array}{c}15.6 \\
\pm 0.31\end{array}$ & $\begin{array}{c}17.6 \pm \\
0.31\end{array}$ & $\begin{array}{c}18.4 \pm \\
0.97\end{array}$ & $\begin{array}{c}19.2 \pm \\
0.15\end{array}$ & $\begin{array}{c}16.2 \pm \\
0.61\end{array}$ & $\begin{array}{c}15.2 \pm \\
0.51\end{array}$ & $\begin{array}{c}15.6 \pm \\
0.31\end{array}$ & $\begin{array}{l}27 \pm \\
0.58\end{array}$ \\
\hline $\begin{array}{l}\text { Staphylococcus } \\
\text { aureus }\end{array}$ & $\begin{array}{c}19.1 \pm \\
0.74\end{array}$ & $\begin{array}{l}18.1 \pm \\
0.55\end{array}$ & $\begin{array}{l}16.7 \pm \\
0.45\end{array}$ & $\begin{array}{c}17.4 \\
\pm 0.42\end{array}$ & $\begin{array}{c}15.8 \pm \\
0.56\end{array}$ & $\begin{array}{c}17.9 \pm \\
0.44\end{array}$ & $\begin{array}{c}17.6 \pm \\
0.31\end{array}$ & $\begin{array}{c}16.1 \pm \\
0.55\end{array}$ & $\begin{array}{l}15.1 \pm \\
0.32\end{array}$ & $\begin{array}{c}15.3 \pm \\
0.55\end{array}$ & $\begin{array}{l}32 \pm \\
0.00\end{array}$ \\
\hline \multicolumn{12}{|c|}{ Gram-negative bacteria } \\
\hline Escherichia coli & $\begin{array}{c}17.8 \pm \\
0.78\end{array}$ & $\begin{array}{c}16.9 \pm \\
0.75\end{array}$ & $\begin{array}{l}17.3 \pm \\
0.60\end{array}$ & $\begin{array}{c}17.7 \\
\pm 0.66\end{array}$ & $\begin{array}{l}18.3 \pm \\
0.85\end{array}$ & $\begin{array}{c}18.1 \pm \\
0.72\end{array}$ & $\begin{array}{c}17.8 \pm \\
0.78\end{array}$ & $\begin{array}{c}16.7 \pm \\
0.40\end{array}$ & $\begin{array}{c}15.1 \pm \\
0.32\end{array}$ & $\begin{array}{c}16.8 \pm \\
0.64\end{array}$ & $\begin{array}{l}25 \pm \\
1.00\end{array}$ \\
\hline $\begin{array}{l}\text { Pseudomonas } \\
\text { aeruginosa }\end{array}$ & $\begin{array}{l}14.4 \pm \\
0.35\end{array}$ & $\begin{array}{c}13.4 \pm \\
0.36\end{array}$ & $\begin{array}{c}12.5 \pm \\
0.69\end{array}$ & $\begin{array}{c}12.1 \\
\pm 0.87\end{array}$ & $\begin{array}{l}12.7 \pm \\
0.85\end{array}$ & $\begin{array}{c}13.5 \pm \\
0.25\end{array}$ & $\begin{array}{l}12.1 \pm \\
0.47\end{array}$ & $\begin{array}{c}11.5 \pm \\
0.15\end{array}$ & $\begin{array}{c}12.8 \pm \\
0.75\end{array}$ & $\begin{array}{l}13.8 \pm \\
0.25\end{array}$ & $\begin{array}{l}20 \pm \\
1.00\end{array}$ \\
\hline $\begin{array}{l}\text { Salmonella } \\
\text { paratyphi }\end{array}$ & $\begin{array}{c}19.2 \pm \\
0.61\end{array}$ & $\begin{array}{c}17.7 \pm \\
0.56\end{array}$ & $\begin{array}{c}17.6 \pm 0.4 \\
9\end{array}$ & $\begin{array}{c}16.8 \\
\pm 0.36\end{array}$ & $\begin{array}{c}18.1 \pm 0.9 \\
3\end{array}$ & $\begin{array}{c}17.9 \pm \\
0.74\end{array}$ & $\begin{array}{c}16.9 \pm 0.7 \\
5\end{array}$ & $\begin{array}{c}14.7 \pm \\
0.35\end{array}$ & $\begin{array}{c}16.5 \pm 0 . \\
35\end{array}$ & $\begin{array}{c}17.3 \pm 0 \\
.59\end{array}$ & $\begin{array}{c}27 \pm 0 \\
58\end{array}$ \\
\hline S. typhi & $\begin{array}{c}16.2 \pm \\
0.87\end{array}$ & $\begin{array}{c}17.1 \pm \\
0.21\end{array}$ & $\begin{array}{l}16.8 \pm \\
0.36\end{array}$ & $\begin{array}{c}16.2 \pm \\
0.81\end{array}$ & $\begin{array}{c}15.4 \pm \\
0.31\end{array}$ & $\begin{array}{c}18.2 \pm \\
0.42\end{array}$ & $\begin{array}{c}17.4 \pm \\
0.36\end{array}$ & $\begin{array}{c}16.9 \pm \\
0.55\end{array}$ & $\begin{array}{c}16.8 \pm \\
0.42\end{array}$ & $\begin{array}{c}16.5 \pm \\
0.35\end{array}$ & $\begin{array}{l}22 \pm \\
0.00\end{array}$ \\
\hline Shigella boydii & $\begin{array}{c}16.8 \pm \\
0.36\end{array}$ & $\begin{array}{c}17.8 \pm \\
0.56\end{array}$ & $\begin{array}{l}17.5 \pm \\
0.31\end{array}$ & $\begin{array}{c}16.5 \pm 0 \\
.35\end{array}$ & $\begin{array}{c}15.4 \pm \\
0.31\end{array}$ & $\begin{array}{c}17.3 \pm \\
0.59\end{array}$ & $\begin{array}{c}17.7 \pm \\
0.56\end{array}$ & $\begin{array}{c}15.9 \pm \\
0.32\end{array}$ & $\begin{array}{c}17.2 \pm 0 . \\
47\end{array}$ & $\begin{array}{l}16.9 \pm \\
0.15\end{array}$ & $\begin{array}{l}27 \pm \\
0.58\end{array}$ \\
\hline Sh. dysenteriae & $\begin{array}{l}16.9 \pm \\
0.45\end{array}$ & $\begin{array}{l}18.1 \pm \\
0.55\end{array}$ & $\begin{array}{l}16.7 \pm \\
0.35\end{array}$ & $\begin{array}{c}15.8 \pm \\
0.56\end{array}$ & $\begin{array}{c}17.2 \pm \\
0.31\end{array}$ & $\begin{array}{c}16.5 \pm \\
0.35\end{array}$ & $\begin{array}{c}17.5 \pm \\
0.29\end{array}$ & $\begin{array}{c}16.1 \pm \\
0.53\end{array}$ & $\begin{array}{c}17.3 \pm \\
0.59\end{array}$ & $\begin{array}{c}13.8 \pm \\
0.71\end{array}$ & $\begin{array}{l}25 \pm \\
0.58\end{array}$ \\
\hline Vibiro mimiscus & $\begin{array}{c}18.5 \pm \\
0.53\end{array}$ & $\begin{array}{c}17.6 \pm \\
0.31\end{array}$ & $\begin{array}{l}16.9 \pm \\
0.15\end{array}$ & $\begin{array}{c}15.8 \pm \\
0.31\end{array}$ & $\begin{array}{l}16.1 \pm \\
0.53\end{array}$ & $\begin{array}{c}17.8 \pm \\
0.36\end{array}$ & $\begin{array}{c}18.1 \pm \\
0.55\end{array}$ & $\begin{array}{c}16.2 \pm \\
0.36\end{array}$ & $\begin{array}{c}14.5 \pm \\
0.40\end{array}$ & $\begin{array}{c}13.8 \pm \\
0.71\end{array}$ & $\begin{array}{l}25 \pm \\
0.58\end{array}$ \\
\hline V. parahemolyticus & $\begin{array}{c}15.8 \pm \\
0.31\end{array}$ & $\begin{array}{l}15.1 \pm \\
0.35\end{array}$ & $\begin{array}{c}15.5 \pm \\
0.74\end{array}$ & $\begin{array}{l}16.1 \pm \\
0.85\end{array}$ & $\begin{array}{c}15.6 \pm \\
0.46\end{array}$ & $\begin{array}{c}16.9 \pm \\
0.15\end{array}$ & $\begin{array}{c}17.9 \pm \\
0.45\end{array}$ & $\begin{array}{c}14.2 \pm \\
0.15\end{array}$ & $\begin{array}{c}15.1 \pm \\
0.45\end{array}$ & $\begin{array}{c}12.2 \pm \\
0.70\end{array}$ & $\begin{array}{l}20 \pm \\
0.58\end{array}$ \\
\hline Fungus & & & & & & & & & & & Gri \\
\hline Aspergillus niger & $\begin{array}{c}14.4 \pm \\
0.42\end{array}$ & $\begin{array}{l}12.2 \pm \\
0.87\end{array}$ & $\begin{array}{l}13.7 \pm \\
0.72\end{array}$ & $\begin{array}{c}13.9 \pm \\
0.57\end{array}$ & $\begin{array}{c}13.5 \pm \\
0.35\end{array}$ & $\begin{array}{c}15.5 \pm \\
0.51\end{array}$ & $\begin{array}{c}14.5 \pm \\
0.36\end{array}$ & $\begin{array}{c}15.4 \pm \\
0.31\end{array}$ & $\begin{array}{c}12.3 \pm \\
0.25\end{array}$ & $\begin{array}{c}13.8 \pm \\
0.71\end{array}$ & $\begin{array}{l}20 \pm \\
0.00\end{array}$ \\
\hline Candida albicans & $\begin{array}{c}15.1 \pm \\
0.91\end{array}$ & $\begin{array}{c}15.6 \pm \\
0.31\end{array}$ & $\begin{array}{l}16.1 \pm \\
0.85\end{array}$ & $\begin{array}{c}14.6 \pm \\
0.64\end{array}$ & $\begin{array}{c}12.5 \pm \\
0.32\end{array}$ & $\begin{array}{c}14.4 \pm \\
0.35\end{array}$ & $\begin{array}{l}13.8 \pm \\
0.35\end{array}$ & $\begin{array}{c}10.2 \pm \\
0.46\end{array}$ & $\begin{array}{c}12.2 \pm \\
0.61\end{array}$ & $\begin{array}{c}11.9 \pm \\
0.61\end{array}$ & $\begin{array}{l}18 \pm \\
0.58\end{array}$ \\
\hline $\begin{array}{l}\text { Saccharomyces } \\
\text { cerevisiae }\end{array}$ & $\begin{array}{c}15.5 \pm \\
0.38\end{array}$ & $\begin{array}{c}12.5 \pm \\
0.32\end{array}$ & $\begin{array}{c}11.9 \pm \\
0.72\end{array}$ & $\begin{array}{c}13.2 \pm \\
0.51\end{array}$ & $\begin{array}{l}12.1 \pm \\
0.74\end{array}$ & $\begin{array}{c}14.1 \pm \\
0.76\end{array}$ & $\begin{array}{c}13.5 \pm \\
0.15\end{array}$ & $\begin{array}{c}13.5 \pm \\
0.15\end{array}$ & $\begin{array}{c}14.2 \pm \\
0.30\end{array}$ & $\begin{array}{c}12.8 \pm \\
0.46\end{array}$ & $\begin{array}{l}19 \pm \\
0.58\end{array}$ \\
\hline
\end{tabular}

$\mathrm{CME}=$ Methanol extract; $\mathrm{HSF}=n$-hexane soluble fraction; $\mathrm{CSF}=$ Carbon tetrachloride soluble fraction; $\mathrm{CHSF}=\mathrm{Chloroform}$ soluble fraction; $\mathrm{AQSF}=$ Aqueous soluble fraction; $\mathrm{PC}=$ Positive control, $\mathrm{Kan}=$ Kanamycin disc and Gri $=$ Griseofulvin disc 
Table 4. $\mathrm{LC}_{50}$ and $\mathrm{LC}_{90}$ values of the crude methanolic extract and its four soluble fractions in brine shrimp lethality bioassay.

\begin{tabular}{|c|c|c|c|c|}
\hline & & $\mathrm{R}^{2}$ & $\mathrm{LC}_{50}(\mu \mathrm{g} / \mathrm{ml})$ & $\mathrm{LC}_{90}(\mu \mathrm{g} / \mathrm{ml})$ \\
\hline \multicolumn{5}{|l|}{ Standard } \\
\hline Vincristine sulfate & $30.799 x+60.645$ & 0.9730 & $0.45 \pm 0.11$ & $10.0 \pm 0.32$ \\
\hline \multicolumn{5}{|l|}{ Samples } \\
\hline \multicolumn{5}{|l|}{ Stem bark } \\
\hline CME & $y=29.398 x+21.323$ & 0.9280 & $9.45 \pm 0.45$ & $216.83 \pm 0.43$ \\
\hline HSF & $y=30.808 x+28.564$ & 0.9573 & $4.96 \pm 0.59$ & $98.66 \pm 0.30$ \\
\hline CSF & $y=24.567 x+45.351$ & 0.7997 & $1.55 \pm 0.30$ & $65.68 \pm 0.23$ \\
\hline CHSF & $y=30.005 x+29.566$ & 0.8400 & $4.79 \pm 0.65$ & $103.31 \pm 0.49$ \\
\hline AQSF & $y=33.829 x+17.790$ & 0.9719 & $8.95 \pm 0.70$ & $136.28 \pm 0.47$ \\
\hline \multicolumn{5}{|l|}{ Leaves } \\
\hline CME & $y=31.413 x+22.810$ & 0.9503 & $7.34 \pm 0.40$ & $137.70 \pm 0.08$ \\
\hline HSF & $y=34.636 x+18.788$ & 0.9378 & $7.96 \pm 0.19$ & $113.77 \pm 0.38$ \\
\hline CSF & $y=28.996 x+25.824$ & 0.9880 & $6.82 \pm 0.67$ & $163.41 \pm 0.22$ \\
\hline CHSF & $y=27.385 x+25.834$ & 0.8243 & $7.63 \pm 0.16$ & $220.35 \pm 0.33$ \\
\hline AQSF & $y=34.232 x+19.292$ & 0.9774 & $7.89 \pm 0.52$ & $116.29 \pm 0.69$ \\
\hline
\end{tabular}

Table 5. Thrombolytic activity (\% clot lysis) of the crude methanolic extract and its four Kupchan fractions.

\begin{tabular}{lc}
\hline & \% of clot lysis \\
\hline Standard & $66.77 \pm 0.61$ \\
SK & \\
Blank & $1.87 \pm 0.38$ \\
Water & \\
Samples & \\
Stem bark & $12.45 \pm 1.31$ \\
CME & $14.27 \pm 0.98$ \\
HSF & $22.75 \pm 0.59$ \\
CSF & $6.34 \pm 0.47$ \\
CHSF & $12.82 \pm 0.35$ \\
AQSF & \\
Leaves & $15.65 \pm 0.94$ \\
CME & $15.33 \pm 0.32$ \\
HSF & $8.02 \pm 1.78$ \\
CSF & $12.19 \pm 1.07$ \\
CHSF & $15.35 \pm 0.35$ \\
AQSF & \\
\hline
\end{tabular}

In the circulatory system vascular blockage, sometimes leading to death is caused by thrombus formation. Thrombolytic agents that include tissue plasminogen activator, urokinase, streptokinase etc. are currently used but these agents are still associated with risk of hemorrhage, anaphylactic reaction and also lack specificity. So, attempts are still ongoing around the world to develop improved thrombolytic agents (Wilson et al., 2008; Rodriguez et al., 2012; Sikder et al., 2012). The crude methanolic extract and its $n$-hexane, carbon tetra chloride, chloroform and aqueous soluble fractions of both the stem bark and leaves of E. fusca revealed low to mild thrombolytic activity (Table 5). The carbon tetrachloride soluble fraction of the stem bark displayed the highest $22.75 \pm 0.59 \%$ clot lysis as compared to $66.77 \pm 0.61 \%$ clot lysis revealed by standard streptokinase. However, as negative control distilled water was used which gave a very minimal percentage of lysis of clot $1.87 \pm 0.38 \%$. The mean difference in clot lysis percentage between positive and negative control was found statistically very significant.

\section{Conclusion}

It is distinctly apparent from the above findings that the leaves and stem bark of E. fusca have significant free radical scavenging ability and 
antioxidant properties. The plant has also exhibited mild to moderate antimicrobial potential, excellent cytotoxic and thrombolytic activities. Therefore, we can conclude that the plant E. fusca is a good candidate for further chemical investigation to isolate the bioactive constituents.

\section{Acknowledgement}

The authors wish to thank Bangladesh National Herbarium, Ministry of Environment and Forest, Dhaka, Bangladesh for identifying the plants and also Biomedical Research Centre, University of Dhaka, Bangladesh and Department of Pharmacy, State University of Bangladesh, Dhaka, Bangladesh for providing some laboratory facilities to carry out some of the experiments.

\section{References}

Agbor, G.A., Vinson, J.A. and Donnelly, P.E. 2014. Folin-Ciocalteau reagent for polyphenolic assay. Int. J. Food Sci. Nutr. Diet.3, 147-156.

Bauer, A.W., Kirby, W.M., Sherris, J.C. and Turck. M. 1966. Antibiotic susceptibility testing by a standardized single disk method. Am. J. Clin. Pathol. 45, 493-496.

Brand-Williams, W., Cuvelier, M.E. and Berset, C.L.W.T. 1995. Use of a free radical method to evaluate antioxidant activity. LWT-FOOD Sci. Technol. 28, 25-30.

Dasuki, U.D. 2001. Medicinal and Poisonous Plants 2, Van Valken-burg J.L.C.H., Bunyapraphatsara N., Backhuys Publishers, Leiden. p. 252-253.

Prasad, S., Kashyap, R.S. Deopujari, J.Y., Purohit, H.J, Taori1, G.M. and Daginawala H.F. 2006. Development of an in vitro model to study clot lysis activity of thrombolytic drugs. Thromb. J. 4(14). doi:10.1186/1477-9560-4-14

Flora of Bangladesh. Accessed on 2020 (http://bnh-flora. gov.bd/species-description/?id=1365)

Gledhill, D. 2008. The Names of Plants. (4th ed.), Cambridge University Press, p. 157.

Karaman, S., Tutem, E., Baskan, K.S. and Apak, R. 2010. Comparison of total antioxidant capacity and phenolic composition of some apple juices with combined HPLC-CUPRAC assay. Food Chem. 120, 1201-1209.

Manilal, A., Sujith, S., Seghal, K.G., Selvin, J. and Shakir, C. 2009. Cytotoxic potentials of red alga, Laurencia brandenii collected from the Indian coast. Global J. Pharmacol. 3, 90-94.
Mazid, M.A., Datta, B.K., Nahar, L. and Sarker, S.D. 2008. Assessment of anti-bacterial activity and brine shrimp toxicity of two Polygonum species. Ars Pharm. 49, 127-134.

Mc Kee, T.C., Bokesch, H.R., Mc Cormick, J.L., Rashid, A., Spielvogel, D., Gustafson, K.R., Alavanja, M.M., Cardelina II, .J.H. and Boyd, M.R. 1997. Isolation and characterization of new anti-HIV and cytotoxic leads from plants, marine and microbial organisms. J. Nat. Prod. 60, 431-438.

Orwa, C., Mutua, A., Kindt, R., Jamnadass, R. and Anthony, S. 2009. Agroforestree Database: a tree reference and selection guide version 4.0. World Agroforestry Center, Kenya. 1.

Perry, L. M. 1980. Medicinal Plants of East and Southeast Asia. MIT Press, Cambridge.

Prieto, P., Pineda, M. and Aguilar, M. 1999. Spectrophotometric quantitation of antioxidant capacity through the formation of a phosphomolybdenum complex: specific application to the determination of vitamin E. Anal. Biochem. 269, 337-341.

Rahman, M.S., Begum, B., Chowdhury, R., Rahman, K.M. and Rashid, M.A. 2008a. Preliminary cytotoxicity screening of some medicinal plants of Bangladesh. Dhaka Univ. J. Pharm. Sci. 7, 47-52.

Ratnasooriya, W.D. and Dharmasiri, M.G.1999. Aqueous extract of Sri Lankan Erythrina indica leaves had sedative but not analgesic activity. Fitoterapia 7, 311-313.

Russo, R. and Baguinon, N.T. 1997. Erythrina fusca Loureiro Fl. Cochinch. 427, 1790.

Rice-Evans, C.A., Miller, N.J. and Paganga, G. 1996. Structure-antioxidant activity relationships of flavonoids and phenolic acids. Free Radical Bio. Med. 20, 933-956.

Rodriguez, A.L., Wojcik, B.M., Wrobleski, S.K., Myers, D.D., Jr., Wakefield, T.W. and Diaz, J.A. 2012. Statins, inflammation and deep vein thrombosis: a systematic review. J. Thromb. Thrombolysis 33, 371-382. https://doi.org/10.1007/s11239-012-0687-9

Ross, S.A., Megalla, S.E., Bishay, D.W. and Awad, A.H. 1980. Studies for determining antibiotic substances in some Egyptian plants. Part I. Screening for antimicrobial activity. Fitoterapia 51, 303-308.

Russo, R. O. and Baguinon N. T. 1997. Erythrina fusca Loureiro. http://www.proseanet.org. [Accessed online 29 March 2020].

Salma, K., Gabr, R. O., Bakr, H. M., Elshishtawy, Ahlam, M. and Taha, S. E. 2017. Botanical and genetic characters of Erythrina neilliicultivated in Egypt. Rev. Bras. Farmacogn. 27, 273-281. 
Sanchez-Moreno, C., Larrauri, J.A. and Saura-Calixto, F. 1998. A procedure to measure the antiradical efficiency of polyphenols. J. Agric. Food Chem. 47, 425-431.

Sikder, M. A. A., Kaisar, M. A., Rashid, M. A., Millat, M. S. and Sultana, A. 2012. In vitromembrane stabilizing activity, total phenolic content, cytotoxic, thrombolytic and antimicrobial activities of Calliandra surinamensis (Wall.). J. Pharmacog. Phytochem. 1, 45-50.

Singapore Botanic Gardens (2013). Lour. https:// florafaunaweb.nparks.gov.sg/specialpages /plant-detail. aspx ?id=2879. [Accessed online 1st July 2019].

Singleton, V.L., Orthofer, R. and Lamuela-Raventos, R.M. 1999. Analysis of total phenols and other oxidation substrates and antioxidants by means of Folin-Ciocalteu reagent. Meth. Enzymol. 299, 152-178.

Skerget, M., Kotnik, P., Hadolin, M., Hras, A.R., Simonic, M. and Knez, Z. 2005. Phenols, proanthocyanidins, flavones and flavonols in some plant materials and their antioxidant activities. Food Chem. 89, 191-198.
Stein, G. 2009. Introduction to the coral trees (Erythrinaspecies). https://davesgarden.com/guides/ articles/view/2594. [Accessed online 29 March 2020].

Kawsar, M.H., Sikder, M.A.A., Rana, M.S., Nimmi, I and Rashid, M.A. 2011. Studies of thrombolytic, antioxidant and cytotoxic properties of two Asteraceous plants of Bangladesh. Bangladesh Pharm. J. 14, 103-106.

Valkenburg, J. L. C. H. and Bunyapraphatsara, N. 2001. Plant resources of South-East Asia: Medicinal and poisonous plants 2. Backhuys Publishers, Netherlands, pp. 252-532.

Wasuwat, S. 1967. A list of Thai Medicinal Plants. ASRCT, Bangkok, p. 22.

Widianto, M. B., Padmawinata, K. and Suhalim, H. 1980. An evaluation of the sedative effect of the seeds of Erythrina fusca Lour. $4^{\text {th }}$ Asian Symposium on Medicinal Plants and Spices, Thailand, p. 147.

Wilson, J. T. and Chaikof, E. L. 2008. Thrombosis and inflammation in intra portal islet transplantation: a review of pathophysiology and emerging therapeutics. J. Diabetes Sci. Technol. 2, 746-759. 